\title{
Research ethics committee audit: differences between committees
}

\author{
M E Redshaw, A Harris, J D Baum Institute of Child Health, University of Bristol
}

\begin{abstract}
The same research proposal was submitted to 24 district health authority (DHA) research ethics committees in different parts of the country. The objective was to obtain permission for a multi-centre research project. The study of neonatal care in different types of unit (regional, subregional and district), required that four health authorities were approached in each of six widely separated health regions in England. Data were collected and compared concerning aspects of processing, including application forms, information required, timing and decision-making. The key finding was that ethics committees received and processed the applications variably, reflecting individual factors and local problems. To improve consensus and facilitate multicentre studies, standard forms and instructions are suggested and the establishment of a national committee or advisory group advocated.
\end{abstract}

\section{Introduction}

Recognition of the role of ethics committees in the area of medical research has grown substantially. ${ }^{1-5}$ The Department of Health (DOH) guidelines ${ }^{6-8}$ emphasise the importance of medical research, the responsibility of the National Health Service (NHS) in supporting research and the role of a local research ethics committee (LREC) in advising on the ethics of research using human subjects. An analysis is reported of data collected prospectively on the submission and processing of a multi-centre study concerned with the organisation and staffing of neonatal care in England. Specific points and general issues arising in the course of submission are presented and discussed.

\section{Methods}

Applications were made to the widely distributed districts in which six regional, six subregional and twelve district neonatal units were located. The research was designed in three stages: general data not

\section{Key words}

Neonatal care; audit; LRECs. requiring ethical permission were to be collected from 56 units; individual data collection on nurses in 24 of these units was planned and parents whose babies had recently been patients in the same 24 units were to be approached. ${ }^{9}$ Parents whose babies had died were to be included. The chairmen, chairwomen or secretaries of 24 district health authority research ethics committees were contacted requesting forms and instructions and ethical applications were made for the second and third stages of the project. Submission and processing took place over a year.

\section{Results}

SUBMISSION

At the time of application ten committees referred to themselves as "ethical" and 14 also used the word "research" in the title. The information requested varied: 16 of the 24 had a specific form for submission; five required that the form be supplemented by a full protocol and the remainder required only a protocol. Additional information on specific points was requested by ten committees and four invited attendance at the relevant meeting.

The length of the form ranged from one to ten pages (median five) and contained between seven and 21 questions (median 13). One contained a total of more than 50 sub-questions. Between one and 19 copies of the application form (median six) were required by the different committees and up to 12 copies of the parents' letters and questionnaires. Letters of local support were requested by nine of the 24 committees, most commonly from hospital consultants in the target hospitals.

The availability and quality of instructions varied: half the committees provided instructions about submission, five by letter and seven on separate sheets. These ranged from two to seven pages and were largely oriented towards applications for clinical trials. All but one of the teaching districts approached sent some instructions, contrasting with the 18 non-teaching districts from whom three sets of formal instructions were received.

PROCESSING

The time required for processing varied. The lag 
between submission and approval ranged from 22 to 298 days, with a mean of 109 days. Meetings were held monthly, bi-monthly, or quarterly. Teaching districts were only slightly more likely to respond and process proposals more quickly than other districts (teaching district range 36-149 days, mean 102 days, compared with a range for other districts of 22-298 days and mean of 111 days). Delays were in some instances the result of consulting parent advisors (representatives of user groups), though for six of the submissions delays were linked to local management, personnel or skill-mix problems, rather than to specific ethical issues arising from the proposed research.

The committees responded variably: 14 gave approval without modification and three rejected the proposal. The three different grounds given for refusal were: that the questionnaires for use with parents were poorly designed; that it might be unethical for a neonatal unit to release the identities of babies to the research team, and that the proposed research conflicted with another ongoing study in the area. Further enquiries and a subsequent site visit could find no evidence for the last of these.

A total of six committees requested minor modifications to the wording of parents' letters, different in each case. Alterations to the subject groups were requested by three committees: two wished the parents whose babies had died to be left out and one requested that no parents take part at all. In all three cases these units were located in district general hospitals. One of those refusing initially, agreed, following procedural changes in the method of contacting parents to involve local medical and nursing staff. Apprehension about parental involvement in the research was the most frequently voiced concern, even from committees giving permission. Five suggested parents should be approached initially via hospital staff, general practitioners or health visitors.

It was calculated that obtaining ethical permission from the 24 district health authorities cost a minimum of $£ 4,000$. This included a total of 280 hours recorded work-time, telephone calls, postage and photocopying, but not overheads. At $£ 166$ per committee the costs were greater than those reported for a study focusing on the Thames regions. ${ }^{10}$

\section{Discussion}

In the process of submission to the 24 committees and in the course of visiting the hospitals involved, a number of specific interrelated issues of general concern emerged.

ETHICAL CONSIDERATIONS AND SCIENTIFIC MERIT According to the DOH guidelines, ${ }^{6}$ the stated concern of a local research ethics committee is with ethics and little direct reference is made to the question of scientific merit. In addition to commenting on ethical aspects of the research some committees sought to advise on the scientific validity and methodology. The use of an unsuitable research design or an ill-conceived hypothesis has ethical implications, but the guidelines do not address the point fully. Whether a committee is to function as an ethics or research committee, or both, requires clarification..$^{4-5}$ Concern has been expressed about the level of local expertise available on the wide range of subjects and scientific issues presented to ethics committees. ${ }^{11}$

For many projects, especially multi-centre studies, the scientific value has been critically appraised prior to submission to individual local research ethical committees. Medical and other authorities in the appropriate fields will have scrutinised the proposal and assessed the value of the projected study. ${ }^{11}$ It would appear wasteful for each local committee to duplicate the process, expecting its local members to repeat the scientific assessment. While comments on study hypotheses, design, and local applicability from experienced members of the committee may contribute to a study being executed successfully, it seems important that the limits of the specific expertise available be recognised.

\section{INDEPENDENCE}

The 1991 Department of Health guidelines state that the members of an LREC are not representatives of the NHS bodies which collaborate in its establishment, nor is the local research ethics committee as a whole an arm of management of any of these bodies. However, much as Moodie and Marshall ${ }^{12}$ have highlighted, when the actual study applications were made, the committees did not appear to be functioning independently and separately from district health authorities. The committees to whom submissions were made gave permission for the study to go ahead directly, and did not appear to pass the decision on to the NHS body concerned for them in turn to communicate to the applicant. Channels of communication are assumed to exist, that are in fact absent. ${ }^{12}$

The question of independence also arises in relation to individuals. Serving committee members give their views on a research proposal, but outside opinion is often sought and those consulted are likely to be clinicians or other NHS employees in the same DHA and often in the same hospital. In seeking advice from specialist referees, it seems important not simply to ask the views of individuals associated with the specific institutions that are involved in a study, directly or indirectly..$^{13}$ Guidance concerning any member of an ethical committee who has an interest which may affect his or her consideration of a particular research proposal is limited and what exactly constitutes "an interest" in this context is not defined. A proposal may be supported or opposed for a wide variety of reasons; the possible effects of ? 
participation, and the expected implications of a study and its findings may affect decision-making. A quarter of the committees in the course of the present submissions appeared to have been influenced in this way. It may be that with research proposals in which local statistics are to be examined or where management practices, working conditions and consumer satisfaction are to be investigated, such factors are more likely to be operative.

\section{LOCALS AND UNKNOWNS}

From the varied responses of the different committees and the way the same proposal was handled it was evident that applications could be subject to differing degrees of scrutiny and even-handedness. Recent and ongoing research projects in the target hospitals visited indicated that proposals from outside a district or region may be at a disadvantage in receiving a more critical appraisal than locally originated studies. In contrast, local applicants' submissions, often from individuals working within the institution, may be less closely examined. In some instances greater weight was given to local opinion and advice rather than cognisance being taken of the views of outsiders, or of the actions of other research ethics committees presented with the same project.

The guidelines make no mention of personal representation to research ethics committees. Some invite researchers to attend the meeting at which their proposal is to be discussed, a request with which local applicants would be better placed to comply. If, as emphasised by Neuberger, ${ }^{4}$ there are benefits to attendance, there may be pressure in this direction in the future. However, with multi-centre studies, using a large number of participating trusts, or where study sites are widely separated and distant from the co-ordinating centre, this is potentially demanding of time and resources. ${ }^{14-15}$ Personal appearance at meetings may also have dubious benefits: individuals in positions of authority locally or those with effective interpersonal skills may obtain permission more easily, without necessarily presenting research that is soundly based in ethical or scientific terms.

\section{DECISION-MAKING}

Delays in processing applications and in informing applicants of decisions may be due to a heavy workload and poorly resourced clerical back-up for research ethics committees. ${ }^{4}{ }^{16}$ However, during the numerous submissions for the study, it appeared that some delays and the small number of rejections were associated with local management crises and personnel problems in the areas of the service to be studied, rather than with inadequacies in the proposed research or availability of secretarial assistance.

\section{CONFIDENTIALITY}

Two points can be made about confidentiality: the first concerns patients and their families. The guidelines which place emphasis on the confidentiality of the committee proceedings, patients' medical records and the data collected on individuals, also direct researchers to seek consent from the health professional concerned in the relevant aspect of care. However, for many patients and their families there are a number of health care professionals who could be consulted and informed, and parents or patients may prefer to express their views in confidence. In the study of neonatal care where an important topic was parental experience and satisfaction with the local care and facilities provided, consulting the professionals involved could have confounded the study itself. Problems may also arise when patients participating in a study are transferred between health districts and the numbers of health care workers involved are increased.

The second point about confidentiality concerns research proposals. Discussion about a proposed project outside the committee room, even among professionals, may affect its validity and success. Research proposals covering areas such as management, aspects of service provision, quality of care, staff health and patient satisfaction are particularly vulnerable in this regard.

\section{RESEARCH INVOLVING HUMAN SUBJECTS}

Research on subjects other than patients or volunteers was not specifically addressed in the instructions to applicants nor in the forms received, and the committees' handling of this aspect of the proposal was variable. The position with regard to medical and nursing staff as study subjects (especially in nonclinical studies) was unclear to some committees, who felt that consideration of this part of the study was not within their remit.

Linked with confusion about the position of staff as subjects, is the question of exactly who is a subject and the role of a research ethics committee when the proposed subjects are neither patients nor volunteers in the conventional sense and the study is not a clinical one. In research projects where surveys or interviews with patients' families or the parents of children who are patients are proposed most ethics committees take on a protective role and extend their responsibilities to include these related individuals. As possibly vulnerable groups, especially where serious illness or death is involved, this seems appropriate, but it is difficult to know how far this should extend and over what period of time. That the protection of "all" research subjects is the responsibility of local research ethics committees appears to be clear, in theory at least. Whether all of those involved in the type of study described would wish to have this degree of protection is an open question.

\section{A MEDICAL ORIENTATION}

Local research ethics committees work within DHAs and largely assess research proposals that are 
designed to address medical or clinical questions. The orientation and background of committee membership is generally medical or clinical. ${ }^{4}$ The proposal forms themselves have been devised with a medical research model in mind and are distinctly oriented towards clinical trials involving the use of drugs, new procedures and medical devices.

The composition of the research ethics committees to which the applications were made is largely unknown. However, as Neuberger reported, ${ }^{4}$ the proportion of lay and non-medical members is still relatively small. Not all that happens in a health care context is medical or clinical and certain aspects of local research ethics committee functioning need to take this into account. Increasingly, research which borders on audit is being carried out on more dynamic aspects of the NHS, including management style and structure, training, work organisation and content. Studies in the fields of psychology, sociology and occupational health are less familiar to research ethics committee members. In these contexts the use of questionnaires and interview schedules as methods of data collection are standard practice. Unfamiliarity with such instruments may result in misunderstandings which contrast with an appreciation of the technical procedures or drug regimes proposed in clinical research.

To deal more effectively with all classes of research proposal and better to inform the process, a more broadly based committee membership is needed, with nurses routinely represented, doctors below consultant level (a mixture of those involved in clinical care and research) included, and other non-medical professionals working in health care, in addition to independent lay persons working outside the NHS. ${ }^{45}$ Where the size of committees becomes unwieldy, co-option may provide for broader representation and flexibility.

\section{MULTI-CENTRE STUDIES}

The role of committees in reviewing protocols for multi-centre trials is one in which the guidelines expect them to face two ways at once. ${ }^{12}$ The recent advice is that each committee is free to arrive at its own decision, but at the same time it is suggested that one district ethical committee be nominated on behalf of them all to consider a particular project. ${ }^{6}$ Networks of co-operating research ethics committees are put forward as a means of facilitating larger scale studies. In practice co-operation and agreement may be difficult to obtain; more than one chairman approached for the present study indicated that his committee would not take any account of the views of other ethics committees, from teaching districts or otherwise. Additionally, many multicentre studies are designed to use sites in geographically separate areas, which in some cases may be deliberately unidentified, and thus a local co-operative strategy would be impracticable or ineffective.

Standard forms, designed for clinical and other research proposals, standard guidelines for applicants, and a central mechanism for obtaining general agreement could resolve the organisational and methodological problems engendered by multiple applications. ${ }^{514} \mathrm{~A}$ central medical ethics committee or advisory group concerned both with general issues and large-scale studies, as discussed by Meade, ${ }^{15}$ Gilbert et al,${ }^{16}$ Neuberger ${ }^{4}$ and Lock, ${ }^{17} 18$ organised with input from the professional bodies associated with the specialties, is a mechanism that could ameliorate the present situation. There is obviously a balance to be struck between the independence required of research ethics committees and the need for well designed, large-scale research studies. In the long term it may be preferable to have a national ethics committee to provide first-stage guidance and advice. Only after these first-stage deliberations would local research ethics committees be involved. Discussion of suggested changes in procedures or design would take place at national level. The points made about independence and members' interests could be thus better addressed and the quality of the planned data collection maintained.

\section{Conclusion}

In submitting the same proposal to 24 widely distributed research ethics committees a wide range of responses were elicited. Methodological changes suggested by some committees, if agreed on a wide scale, would have substantially affected the design, data collection and possibly the validity of the study. The process and variation described were not unique to this study. ${ }^{1 .+19}$ Obtaining ethics permission was expensive in terms of time and effort on the part of the research team and the committees processing the submissions. The timing of data collection and analysis was affected by the cumbersome and unwieldy nature of the submission process and the multiple applications, like those required of other researchers, used resources that it was felt could have been directed elsewhere. ${ }^{1+20}$

There is clearly a balance to be sought between facilitating ethical research and safeguarding the interests of research subjects and preventing unethical studies from taking place. The need for adequate administrative support and training for those involved has been emphasised ${ }^{45}$ and, while streamlining of submission procedures and ethics committee organisation may be in hand currently, it seems unlikely that delays and variations of the kind encountered will disappear in the near future. If research and development is to be considered an integral part of health care, then so is the ethical review of research proposals, and like other parts of the health care system LRECs should expect to be audited. ${ }^{15}$ As a result of this experience of the functioning of ethics committees it is recommended that standard forms, instruction and information sheets be agreed and utilised nationally. There would also 
be some advantage in establishing a national committee or advisory group concerned with general ethical principles, scientific merit and multi-centre trials. ${ }^{114}$ Central processing and independent expert opinion in the appropriate fields could reduce the workload for LRECs and researchers alike, while still endorsing the principle that a locally based committee should retain the right of veto and the ultimate responsibility for safeguarding the rights of the individuals who are the subjects of such research.

\section{Acknowledgments}

The research project was commissioned and funded by the Department of Health, but the views expressed are those of the authors alone. We thank Dr Neil Marlow, Dr Karen Thorpe and Dr Theresa Marteau for their comments and the other members of the Project Advisory Group.

$M E$ Redshaw, PhD, A Harris, RN, and $\mathcal{f} D$ Baum, $M A, M S c, M D, F R C P$, are, respectively, Research Fellow, Research Associate and Professor of Child Health at the Institute of Child Health, the University of Bristol.

\section{References}

1 Department of Health and Social Security. Guidelines for research ethics committees. London: DHSS, 1975.

2 Department of Health and Social Security. Local research ethics committees: draft health circular. London: DHSS, 1989.

3 Royal College of Physicians. Guidelines on the practice of ethics committees in medical research involving human subjects. London: RCP, 1990.

4 Neuberger J. Ethics and health care: the role of research ethics committees in the United Kingdom. Research report no 13. London: Kings Fund, 1992.
5 Moran J. Local research ethics committees. Report of the 2nd national conference. Fournal of the Royal College of Physicians. 1992; 26, 4: 423-31.

6 Department of Health. Local research ethics committees. HSG (91) 5, August. London: DOH, 1991.

7 Smith J. New guidelines for British ethics committees. British Medical fournal. 1991; 303: 488.

8 Moodie P. The role of local research ethics committees: maintaining the pressure for improvement. British Medical fournal. 1992; 304: 1129-30.

9 Redshaw M, Harris A, Ingram J. Delivering neonatal care: the neonatal unit as a working environment. 1995, London: HMSO, 1996.

10 Berry T, Ades TE, Peckham CS. Too many ethical committees. British Medical fournal. 1990; 301: 1274.

11 Penn ZT, Steer P. Local research ethics committees: hindrance or help? British fournal of Obstetrics and Gynaecology. 1995; 102: 1-2.

12 Moodie P, Marshall T. Guidelines for local research ethics committees. British Medical fournal. 1992; 304: 1293-5.

13 Harding T, Ummel M. Evaluating the work of ethical review committee: an observation and a suggestion. fournal of Medical Ethics. 1989; 15: 191-4.

14 Middle C, Johnson A, Petty T, Sims L, Macfarlane A. Ethics approval for a national postal survey: recent experience. British Medical Fournal. 1995; 311: 659-60.

15 Meade TW. The trouble with ethics committees. Fournal of the Royal College of Physicians. 1984; 28: 102-4.

16 Gilbert C, Fulford KWM, Parker C. Diversity in the practice of district ethics committees. British Medical fournal. 1989; 299: 1437-9.

17 Lock S. Monitoring research ethical committees. British Medical fournal. 1990; 300: 51-2.

18 Lock S. Towards a national bioethics committee. British Medical fournal. 1990; 300: 1149-50.

19 Garfield P. Cross district comparison of applications to research ethics committees. British Medical fournal. 1995; 311: 660-1.

20 While AE. Ethics committees: impediments to research or guardians of ethical standards? British Medical fournal. 1995; 311: 661.

\section{News and notes}

\section{Care of the Terminally Ill}

The 11th International Congress of the Terminally Ill will be held from the 7th to the 11 th of September this year at the Palais des Congres, Montreal, Canada.

Topics include: When young doctors care for the dying; Children and dying: rediscovering what really matters; Conjugal bereavement; Dealing with our guilt, and The pursuit of inner health.

For further information please contact: The 11th International Congress Secretariat, Events International Meeting Planners Inc, 759 Victoria Square, Suite 700, Montreal (Qc) H2Y 2J7. Telephone: (514) 286-0855; fax: (514) 286-6066. 